\title{
Seismic wave attenuation and dispersion induced by fluid flow within various cracks and a small amount of bubbly fluid
}

\author{
Yu Chen ${ }^{1}$, Zhaoyun Zong*,1, Chongpeng Huang ${ }^{1}$, Shuan Zhang ${ }^{1}$ \\ (1) China University of Petroleum, Qingdao, Shandong, China
}

Article history: received July 1, 2019; accepted January 13, 2020

\begin{abstract}
Hydrocarbon reservoirs usually contain pores and various cracks, which may contain a small amount of bubble fluid. In this study, based on the wave theory of basic porous medium, the influence of various factors on fluid pressure are investigated, and then the stress-strain relationship and Lagrange's equations with a dissipation function is utilized to derive the elastic wave equation of porous medium containing various cracks and a small amount of bubbly fluid initially. This elastic wave equation describes the influence of squirt flow induced by various cracks on seismic wave attenuation and dispersion, and the influence of the bubble linear vibration on seismic wave attenuation and dispersion effectively. Then, the seismic wave attenuation and dispersion of a given model is estimated and the matching of rock physics parameters are obtained in different frequency bands. The numerical results illustrate that the proposed approach is compatible with previous theory to explain the mechanics of the seismic wave attenuation and dispersion induced by fluid flow and can better describe the propagation of elastic waves in actual rock medium especially for the mechanics of the seismic wave attenuation and dispersion induced by fluid flow within various cracks and a small amount of bubble fluid.
\end{abstract}

Keywords: Attenuation, Dispersion, Crack, Bubbly Fluid, WIFF.

\section{Introduction}

Seismic waves in the Earth's medium possess attenuation and dispersion characteristic over a wide frequency range [Aki and Richards, 1980]. Numerous physical process mainly including elastic process and inelastic dissipation, may result to the attenuation and dispersion of seismic wave. Exploration geophysicists are particularly interested in the inelastic attenuation and dispersion due to the presence of fluid in the pores of the rock [Kumar et al., 2019] by the mechanism wave-induced fluid flow (WIFF) [Müller et al., 2010] including macroscopic scale, microscopic scale and mesoscopic scale.

WIFF occurs from macroscopic flow caused by pressure gradients at the wavelength scale. Viscous-inertial attenuation and dispersion due to macroscopic flow is proposed by Biot's theory [Biot, 1956a, 1956b, 1962; Wang et al., 1990; Pride, 2005; Mavko et al., 2009]. In accordance with these studies, macroscopic flow attenuation well exceeds the seismic frequency band. 


\section{Yu Chen et al.}

On the other hand, WIFF occurs from microscopic flow (squirt flow) caused by pressure gradients at the microstruture scale. According to the studies focused on microscopic flow [Mavko and Nur, 1979; Murphy et al., 1986; Mavko et al., 1991; Parra, 1997; Dvorkin et al., 1995], the microscopic flow generally considered to be important at petrophysical frequencies but may also contribute to seismic and logging frequencies. Among these studies, one of typical theories is BISQ theory, which defines the squirt length ' $\mathrm{R}$ ' to analyze the squirt flow [Dvorkin and Nur, 1993; Dvorkin et al., 1994]. However, the slow P wave of BISQ theory has a large attenuation in the low frequency band and is incompatible with the real situation of elastic wave attenuation [Tang, 2011]. Therefore, coin-type and pinch-type crack is respectively introduced into porous medium to make up for this deficiency [Tang, 2011; Tang et al., 2012]. Furthermore, coin-type and pinch-type crack is simultaneously introduced into porous medium to describe seismic wave dispersion and attenuation [Wu et al., 2015].

WIFF can also occur from mesoscopic flow caused by spatial variations at the scale of much larger than microstruture but much less than the wavelength. Mesoscopic flow can cause attenuation and dispersion over a wide range of frequencies, especially significant in seismic bands. [Pride et al., 2004]. Two important models about attenuation and dispersion have been proposed, including the double-porosity model and patchy-saturated model. The double-porosity model is proposed by Pride and Berryman [2003a, 2003b] and extended to many special situations [Ba et al., 2011, 2017; Sun et al., 2018; Zhao et al., 2018; Zhang et al., 2019]. The patchy-saturated model is first proposed by White [1975] and developed by Dutta and Odé [1979a, 1979b] and Vogelaar et al. [2007]. However, on the basis of White's model, when the gas content is small, the presence of gas has little effect on the propagation of seismic waves, which does not conform to bubble vibration theory given by Keller and Miksis [1980]. Therefore, the elastic wave theory of quantifying the attenuation of a small amount of bubble fluid is established [Wang et al., 2018].

Up to now, the model that considers mesoscopic flow, a small amount of bubble fluid and microscopic flow simultaneously is still challenging. Therefore, in this study, we propose a model, a porous medium containing various cracks and a small amount of bubble fluid.

Firstly, we assume that the underground porous medium contains both coin-type, pinch-type cracks and a small amount of bubble fluid. Then under the framework of Biot theory, considering the influence of local flow and bubble linear vibration on seismic wave dispersion and attenuation, we establish an elastic wave equation. Through calculation and analysis, this new theory not only retains the basic characteristics of the previous theory, but also greatly improves the ability to predict and simulate the propagation of elastic waves in porous medium.

\section{The influence of various factors on fluid pressure}

\subsection{Review of the influence of various cracks on fluid pressure}

The fluid pressure of porous medium is [Biot, 1956],

$$
P=-\frac{(\alpha \nabla \cdot \boldsymbol{u}+\nabla \cdot \boldsymbol{w})}{\beta}
$$

where, $\alpha=1-\frac{K_{d}}{K_{s}}, \beta=\frac{(\alpha-\phi)}{K_{s}}+\frac{\phi}{K_{f}}, \boldsymbol{w}=\phi(\boldsymbol{U}-\boldsymbol{u})$, and, $\boldsymbol{u}, \boldsymbol{U}$ is the solid displacement and fluid displacement, respectively. $K_{d}, K_{s}, K_{f}$, are the skeleton, solid, fluid bulk modulus, respectively. $\phi$ is the porosity of the rock.

Under the assumption that coin-type cracks and pinch-type cracks in a porous medium are randomly oriented in the three-dimensional space, we involve cracks into the porous rock, which cause the "squirt flow" between cracks and pores (as shown in Figure 1). Moreover, in a unit rock volume the increase of fluid dilatation due to local flow into pore space is $q_{i}$. The pore pressure in turn is changed to accommodate the added fluid content, $\phi q_{i}$. $i=1,2$ represent coin-type crack and pinch-type crack, respectively. And the fluid pressure is [Wu et al., 2015],

$$
P=-\frac{\left(\alpha \nabla \cdot \boldsymbol{u}+\nabla \cdot \boldsymbol{w}+\phi q_{1}+\phi q_{2}\right)}{\beta}
$$




\section{Attenuation within cracks and bubbly fluid}

Using $q_{1}=S_{1}(\omega) P_{f} / \phi$ and $q_{2}=S_{2}(\omega) P_{f} / \phi$ to characterize the squirt flow influence induced by coin-type crack and pinch-type crack on the pore pressure yields,

$$
P=-\frac{(\alpha \nabla \cdot \boldsymbol{u}+\nabla \cdot \boldsymbol{w})}{\beta+S_{1}(\omega)+S_{2}(\omega)}
$$

The specific expression of $S_{i}(\omega)$ is given in Appendix A.

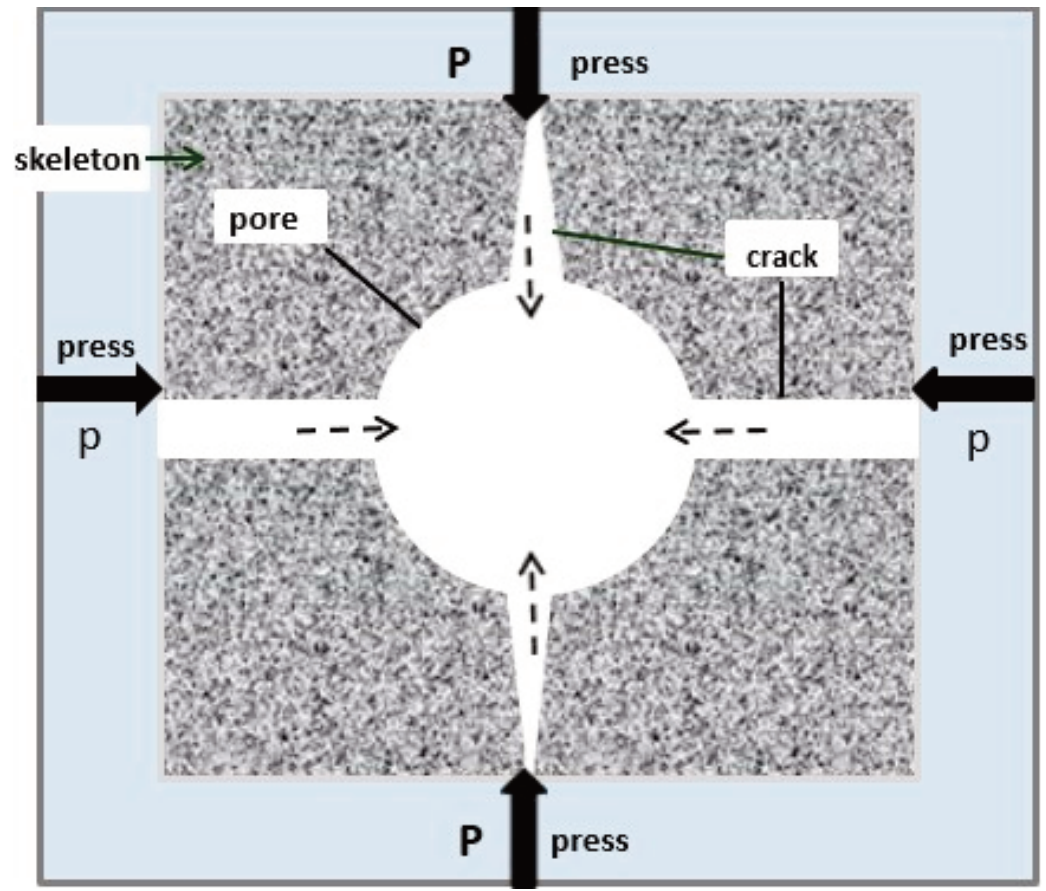

Figure 1. The porous medium containing different kinds of cracks

\subsection{The influence of bubble fluid on fluid pressure}

In addition to the existence of cracks, there is always a small amount of bubble fluid in the underground porous medium (as shown in Figure 2). Therefore, it is necessary to consider the attenuation caused by a small amount of bubble fluid. Following the method to research the attenuation caused by a small amount of bubble fluid, the bubble volume fraction $\phi_{g}$ can be expressed as,

$$
\phi_{g}=\frac{V_{g}}{V_{k}}
$$

where $V_{g}$ is the content of bubbles per unit pores, $V_{g}=4 \pi a^{3} N / 3$, R is the instantaneous radius of the bubble, $N$ is the number of bubbles in the unit volume, $V_{k}$ is the volume of unit pore, $V_{k}=4 \pi a^{3} \mathrm{~N} / 3$ and $a$ is pore radius.

The relationship between the bubble volume fraction $\phi_{g}$ and the instantaneous radius $R$ of the bubble is

$$
\phi_{g}=\frac{N R^{3}}{a^{3}}
$$

Introducing bubbly fluid into the porous rock with the following hypothesis including the bubbles are the same 


\section{Yu Chen et al.}

size and evenly distributed in the fluid, the gas in the bubble is the ideal gas, ignoring the influence of the vapor inside the bubble, the gas and liquid are mutually incompatible and bubble fluid's contribution to the fluid modulus $K_{f}$ could be negligible for its little volume.

Using the commander bubble linear vibration theory [Wang et al., 2018], the fluid pressure is

$$
P=-\frac{(\alpha \nabla \cdot \boldsymbol{u}+\nabla \cdot \boldsymbol{w})}{\beta+\xi}
$$

with

$\xi=\frac{3 N R_{0}}{\rho_{w} a^{3}\left(\omega_{0}^{2}-\omega^{2}+2 i b_{0} \omega\right)} \phi$

$\omega_{0}^{2}=\frac{P_{i n 0}}{\rho_{w} R_{0}^{2}}\left(R e \theta-\frac{2 \sigma}{R_{0} P_{i n 0}}\right)$

$b_{0}=\frac{2 \eta}{\rho_{w} R_{0}^{2}}+\frac{\omega^{2} R_{0}}{2 c}+\frac{P_{i n 0}}{2 \rho_{w} \omega R_{0}^{2}} \operatorname{Im} \theta$

$\theta=\frac{3 \gamma}{1-3(\gamma-1) i \chi\left[(i / \chi)^{1 / 2} \operatorname{coth}(i / \chi)^{1 / 2}-1\right]}$

$\chi=\frac{D}{\omega R_{0}^{2}}$

where $R_{0}$ is the initial radius of the bubble, $P_{i n 0}$ is the pressure inside the bubble at steady state, and $P_{i n 0}=P_{\infty}+2 \sigma / R$ from the boundary condition of the bubble, $N$ is the number of bubbles in the unit volume, $\sigma$ is the surface tension coefficient of the gas-liquid interface, $\eta$ is the pore fluid viscosity coefficient, $D$ is the thermal diffusivity of the gas and $\gamma$ is the specific heat of the gas.

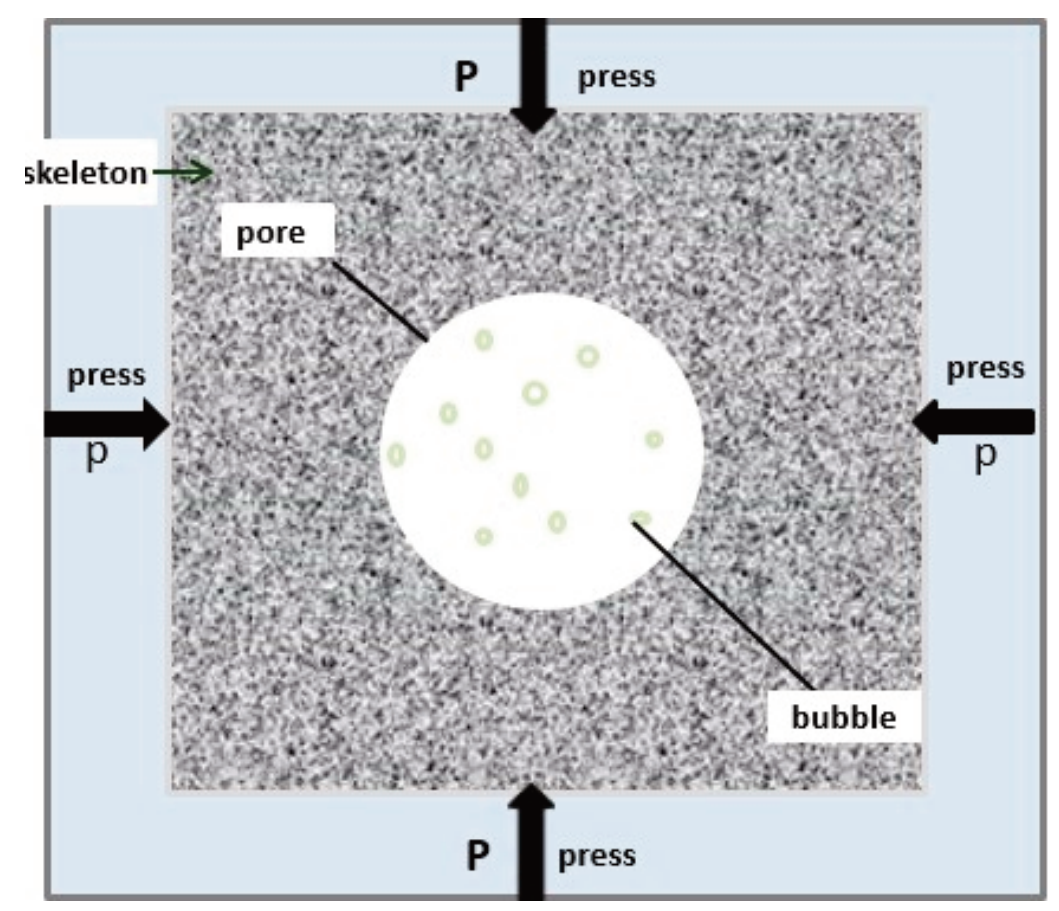

Figure 2. The porous medium containing a small amount of bubble fluid. 


\subsection{The influence of various cracks and bubble fluid on fluid pressure}

In complex underground porous medium, there may be cases where a small amount of bubble fluid is present in mixed cracks, which means the bubble fluid and various cracks are introduced into the porous rock simultaneously. In such case, two mechanisms affect fluid pressure (as shown in Figure 3). We assume that these two factors act independently and do not interfere with each other, and the fluid pressure is

$$
P=-\frac{(\alpha \nabla \cdot \boldsymbol{u}+\nabla \cdot \boldsymbol{w})}{\beta+\xi+S_{1}(w)+S_{2}(w)}
$$

where $\alpha=1-\frac{K_{d}}{K_{s}}, \beta=\frac{(\alpha-\phi)}{K_{s}}+\frac{\phi}{K_{f}}, \boldsymbol{w}=\phi(\boldsymbol{U}-\boldsymbol{u}), \xi=\frac{3 N R_{0}}{\rho_{w} a^{3}\left(\omega_{0}^{2}-\omega^{2}+2 i b_{0} \omega\right)} \phi \cdot \boldsymbol{u}, \boldsymbol{U}$ is the solid displacement and fluid displacement, respectively. $K_{d}, K_{s}, K_{f}$ are the skeleton, solid, fluid bulk modulus, respectively. $\phi$ is the porosity of the rock. The specific expression of $S_{i}(\omega)$ is also given in Appendix A.

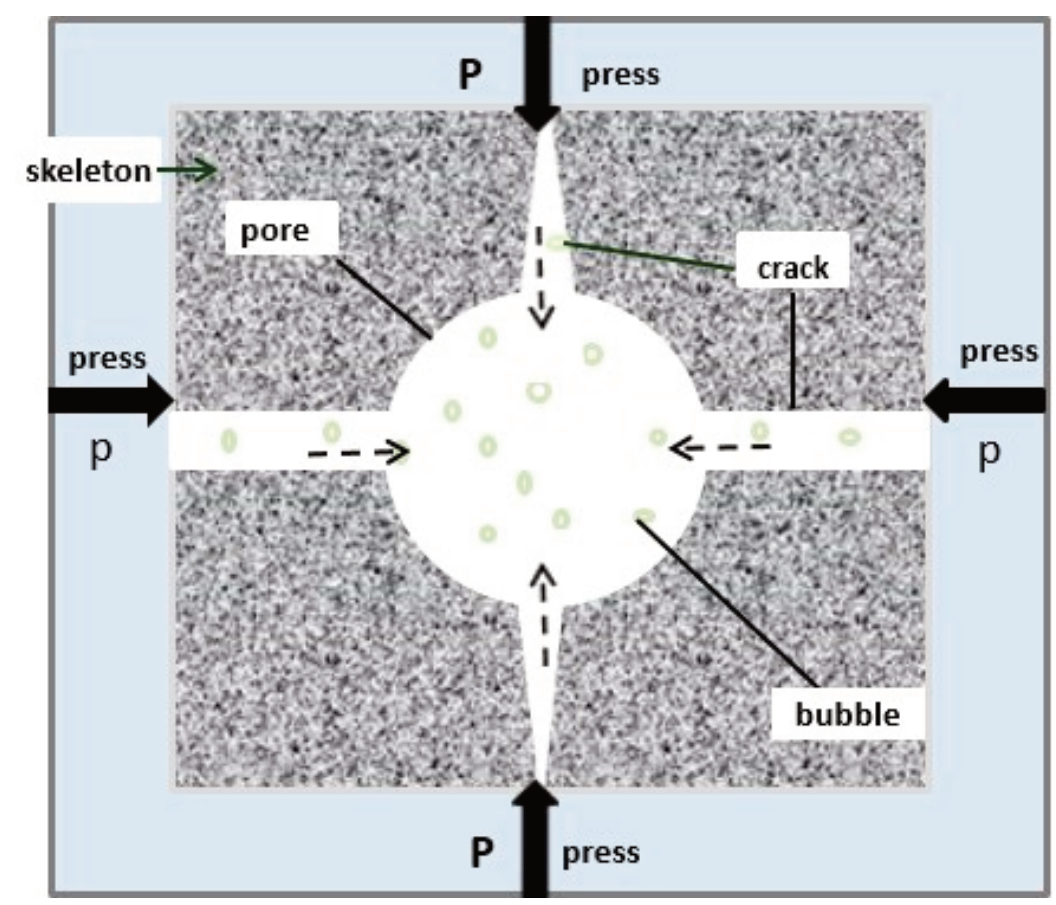

Figure 3. The porous medium containing different kinds of cracks and a small amount of bubble fluid.

\section{Elastic wave equations of porous medium containing various cracks and a small amount of bubbly fluid}

Under the framework of Biot theory, the stress-strain relationship of porous medium containing various cracks and a small amount of bubbly fluid is,

$$
\begin{gathered}
\sigma_{i j}=2 N \varepsilon_{i j}+A \varepsilon \delta_{i j}+Q \zeta \delta_{i j} \\
S_{i j}=Q \varepsilon \delta_{i j}+R \zeta \delta_{i j}
\end{gathered}
$$

where $\delta_{i j}$ is the Kronecker's symbol, $\sigma_{i j}$ is the solid phase stress, $S_{i j}$ is the fluid phase stress, $S=\phi P$. $\varepsilon_{i j}$ is the solid phase strain, $\varepsilon=\nabla \cdot \boldsymbol{u} . \zeta$ is the fluid phase strain and $\zeta=\nabla \cdot \boldsymbol{U}$.

Furthermore, dynamic equations for porous medium containing various cracks and a small amount of bubble fluid derived from Lagrange's equations with a dissipation function [Biot, 1956] are, 


\section{Yu Chen et al.}

$$
\begin{gathered}
\nabla \cdot \sigma=\frac{\partial^{2}}{\partial t^{2}}\left(\rho_{11} \boldsymbol{u}+\rho_{12} \boldsymbol{U}\right)+b \frac{\partial}{\partial t}(\boldsymbol{u}-\boldsymbol{U}) \\
-\phi \nabla \cdot P=\frac{\partial^{2}}{\partial t^{2}}\left(\rho_{12} \boldsymbol{u}+\rho_{22} \boldsymbol{U}\right)-b \frac{\partial}{\partial t}(\boldsymbol{u}-\boldsymbol{U})
\end{gathered}
$$

And the fluid pressure is,

$$
P=\frac{(\alpha \nabla \cdot \boldsymbol{u}+\nabla \cdot \boldsymbol{w})}{\beta+\xi+S_{1}(\omega)+S_{2}(\omega)}
$$

Combining equation (8), (9) and (10), the elastic wave equation of porous medium containing various cracks and a small amount of bubbly fluid can be expressed as,

$$
\begin{gathered}
N \nabla^{2} \boldsymbol{u}+\nabla[(A+N) \varepsilon+Q \zeta]=\frac{\partial^{2}}{\partial t^{2}}\left(\rho_{11} \boldsymbol{u}+\rho_{12} \boldsymbol{U}\right)+b \frac{\partial}{\partial t}(\boldsymbol{u}-\boldsymbol{U}) \\
\nabla(Q \varepsilon+R \zeta)=\frac{\partial^{2}}{\partial t^{2}}\left(\rho_{12} \boldsymbol{u}+\rho_{22} \boldsymbol{U}\right)-b \frac{\partial}{\partial t}(\boldsymbol{u}-\boldsymbol{U})
\end{gathered}
$$

with

$$
\begin{aligned}
& \rho_{11}+\rho_{12}=(1-\phi) \rho_{s}, \rho_{12}+\rho_{22}=\phi \rho_{f}, \rho_{12}=\{1-[1+r(1 / \phi-1)]\} \phi \rho_{f} \\
& b=\eta \phi^{2} \sqrt{1}+i \omega / 2 \omega_{B} / \kappa, \omega_{B}=\frac{\phi \eta}{k \zeta \rho_{f}}, N=\mu, P=A+2 N \\
& P=K_{d}+4 \mu / 3+(\alpha-\phi)^{2} /\left[\beta+\xi+s_{1}(w)+s_{2}(w)\right] \\
& Q=\phi(\alpha-\phi) /\left[\beta+\xi+s_{1}(w)+s_{2}(w)\right] \\
& R=\phi^{2} /\left[\beta+\xi+s_{1}(\omega)+s_{2}(\omega)\right] \\
& \xi=\frac{3 N R_{0}}{\rho_{w} a^{3}\left(\omega_{0}^{2}-\omega^{2}+2 i b_{0} \omega\right)} \phi \\
& \omega_{0}^{2}=\frac{P_{i n 0}}{\rho_{w} R_{0}^{2}}\left(R e \theta-\frac{2 \sigma}{R_{0} P_{i n 0}}\right) \\
& b_{0}=\frac{2 \eta}{\rho_{w} R_{0}^{2}}+\frac{\omega^{2} R_{0}}{2 c}+\frac{P_{i n 0}}{2 \rho_{w} \omega R_{0}^{2}} \operatorname{Im} \theta \\
& \theta=\frac{D}{1-3(\gamma-1) i \chi\left[(i / \chi)^{1 / 2} \operatorname{coth}(i / \chi)^{1 / 2}-1\right]} \\
& \chi R_{0}^{2}
\end{aligned}
$$

where $\boldsymbol{u}, \boldsymbol{U}$ is the solid displacement and fluid displacement, respectively, $\mu$ is the shear modulus, $K_{d}, K_{s}, K_{f}$ are the skeleton, solid, fluid bulk modulus, respectively, $P, Q, R$ are the modulus, respectively, $\rho_{s}$ and $\rho_{f}$ are solid density and fluid density, respectively, $\eta$ and $\kappa$ are the viscosity coefficient and permeability of the fluid, respectively, $\omega$ is the circular frequency and $\omega_{B}$ is the Biot characteristic frequency, $\omega_{0}$ is the resonance frequency, $\alpha$ is the Biot coefficient, ai s pore radius. 
Attenuation within cracks and bubbly fluid

\begin{tabular}{|c|c|c|c|c|c|}
\hline Parameter & Figure 4 & Figure 5 & Figure 6 & Figure 7 & Figure 8 \\
\hline$\phi$ & 0.3 & 0.3 & $0.2-0.3$ & 0.3 & 0.3 \\
\hline$\kappa(\mathrm{D})$ & 1 & 1 & 1 & $1-10$ & 1 \\
\hline$\eta(\mathrm{cp})$ & 0.006 & 0.006 & 0.006 & 0.006 & 0.006 \\
\hline$\rho_{\mathrm{s}}\left(\mathrm{kg} / \mathrm{m}^{3}\right)$ & 2650 & 2650 & 2650 & 2650 & 2650 \\
\hline$\rho_{\mathrm{f}}\left(\mathrm{kg} / \mathrm{m}^{3}\right)$ & 1000 & 1000 & 1000 & 1000 & 1000 \\
\hline$\mu(\mathrm{GPa})$ & 5 & 5 & 5 & 5 & 5 \\
\hline $\mathrm{K}_{\mathrm{s}}(\mathrm{GPa})$ & 36 & 36 & 36 & 36 & 36 \\
\hline $\mathrm{K}_{\mathrm{d}}(\mathrm{GPa})$ & 6 & 6 & 6 & 6 & 6 \\
\hline $\mathrm{K}_{\mathrm{f}}(\mathrm{GPa})$ & 2.25 & 2.25 & 2.25 & 2.25 & 2.25 \\
\hline$\varepsilon_{1}$ & 0.0025 & 0.0025 & 0.0025 & 0.0025 & 0.0025 \\
\hline$\gamma_{1}$ & 0.0020 & 0.0020 & 0.0020 & 0.0020 & 0.0020 \\
\hline$\varepsilon_{2}$ & 0.0025 & 0.0025 & 0.0025 & 0.0025 & 0.0025 \\
\hline$\gamma_{2}$ & 0.0015 & 0.0015 & 0.0015 & 0.0015 & 0.0015 \\
\hline$\phi_{\mathrm{g}}$ & $10^{-4}$ & $0-10^{-4}$ & $10^{-4}$ & $10^{-4}$ & $10^{-4}$ \\
\hline $\mathrm{R}_{0}(\mathrm{~m})$ & $2.0 \times 10^{-6}$ & $2.0 \times 10^{-6}$ & $2.0 \times 10^{-6}$ & $2.0 \times 10^{-6}$ & $2.0 \times 10^{-6}$ \\
\hline $\mathrm{D}\left(\mathrm{m}^{2} / \mathrm{s}\right)$ & $2.4 \times 10^{-5}$ & $2.4 \times 10^{-5}$ & $2.4 \times 10^{-5}$ & $2.4 \times 10^{-5}$ & $2.4 \times 10^{-5}$ \\
\hline$\sigma(\mathrm{N} / \mathrm{m})$ & $72.25 \times 10^{-3}$ & $72.25 \times 10^{-3}$ & $72.25 \times 10^{-3}$ & $72.25 \times 10^{-3}$ & $72.25 \times 10^{-3}$ \\
\hline $\mathrm{P}_{\infty}(\mathrm{Pa})$ & $1.0 \times 10^{5}$ & $1.0 \times 10^{5}$ & $1.0 \times 10^{5}$ & $1.0 \times 10^{5}$ & $1.0 \times 10^{5}$ \\
\hline$\Gamma$ & 1.4 & 1.4 & 1.4 & 1.4 & 1.4 \\
\hline
\end{tabular}

Table 1. Parameter values.

\section{Numerical calculation results and analysis}

We compute the seismic wave attenuation and dispersion for porous medium containing various cracks and a small amount of bubbly fluid using proposed theory and method in this study. The calculation formula is shown in appendix B. Table 1 gives the model parameters.

\subsection{Comparison of the theory of the proposed with previous ones}

Figure 4 (a) and (b) display the dispersion and attenuation for the fast $\mathrm{P}$ wave for Biot theory, porous medium containing coin-type crack and pinch-type crack, porous medium containing a small amount of bubbly fluid and the proposed theory respectively in the frequency range of 10 to $10^{7} \mathrm{~Hz}$ with logarithmic scale. It can be obviously observed from Figure 4(a) that in the proposed model, there is a significant dispersion of fast P wave velocity from low frequency to high frequency. It also can be clearly observed from Figure 4(a) that in the proposed model, the attenuation parameter curve of the seismic wave presents "multiple peaks". Those peaks are induced by squirt flow caused by coin-type crack, squirt flow caused by pinch-type crack, "Biot flow" attenuation mechanism and bubble linear vibration, corresponding to the peak of Cracks curve, Biot curve and Bubbly fluid curve.

Figure 4 (c) and (d) display the dispersion and attenuation for the slow $\mathrm{P}$ wave for Biot theory, porous medium containing coin-type crack and pinch-type crack, porous medium containing a small amount of bubbly fluid and proposed theory respectively in the frequency range of 10 to $10^{7} \mathrm{~Hz}$ with the logarithmic scale. At low frequency, the speed of slow $\mathrm{P}$ waves approach zero, meanwhile the speed increases with frequency. The slow $\mathrm{P}$ wave speed given by the proposed theory is lower than the speed of the Biot theory and unstable in the frequency of $\mathrm{Hz}$, 


\section{Yu Chen et al.}

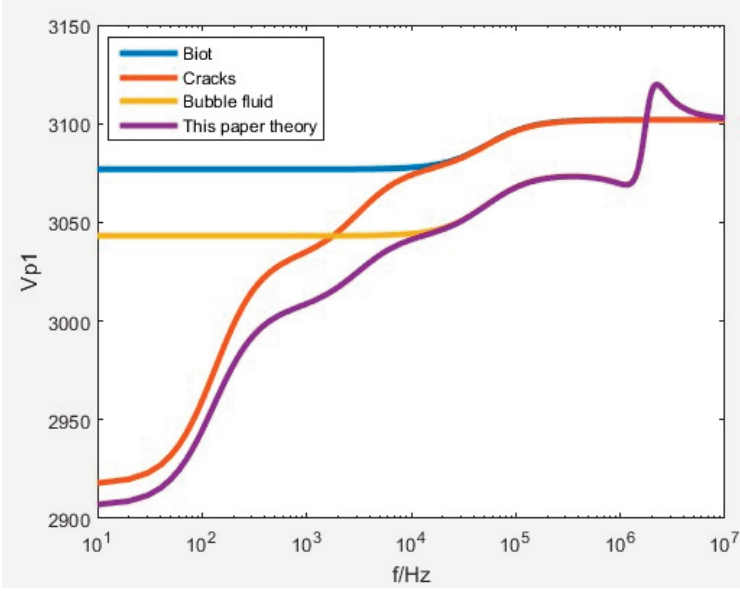

a)

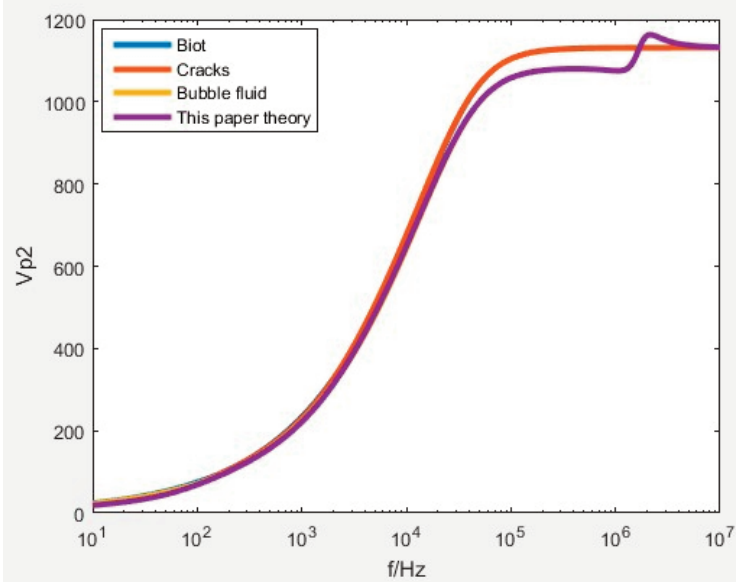

c)

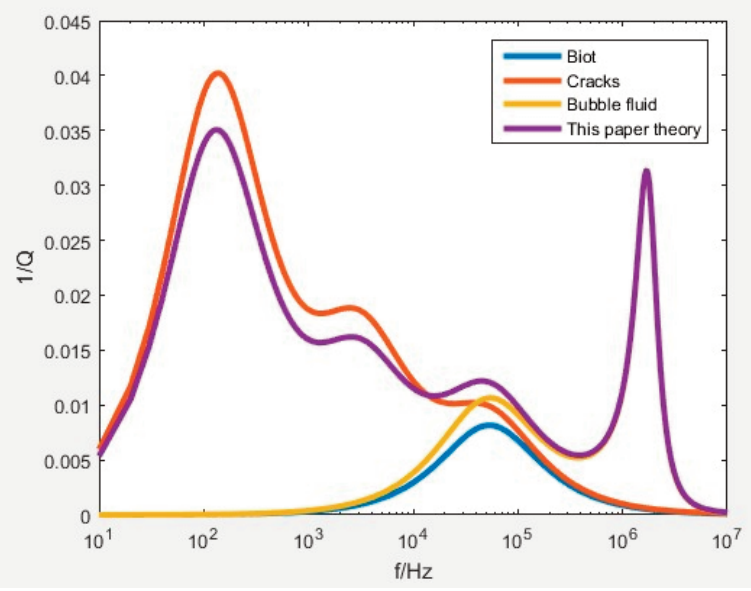

b)

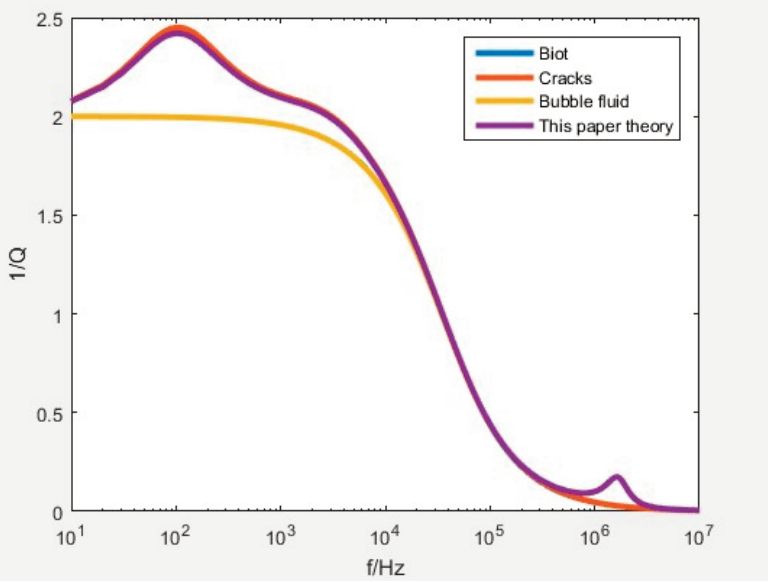

d)

Figure 4. The attenuation and dispersion of the fast and slow $\mathrm{P}$ waves for different theories. The dispersion velocity (a) and attenuation (b) for the fast $\mathrm{P}$ wave, the dispersion velocity (c) and attenuation (d) for the slow $\mathrm{P}$ wave.

which is resulted in the additional attenuation caused by the squirt flow and bubble linear vibration. Moreover, the low-frequency attenuation characteristics of the slow $\mathrm{P}$ wave are similar to Biot's theory slow $\mathrm{P}$ wave attenuation characteristics, with $1 / \mathrm{Q}$ value approaching around two in the low-frequency limit.

From those, we can discern that the proposed model is compatible with the characteristics of previous theory and can better describe the propagation of seismic waves in underground porous medium.

\subsection{Comparison of the proposed model with patchy-saturated model}

Figure 5(a) and (b) display the dispersion and attenuation for the $\mathrm{P}$ wave for patchy-saturated model [Vogelaar et al., 2010] and the proposed model in the frequency range of $10 \mathrm{to} 10^{7} \mathrm{~Hz}$ with logarithmic scale. It can be clearly seen from Figure 5(b) that in the two models, the attenuation parameter curve of the seismic wave presents "multiple peaks". In the patchy-saturated model, those peaks are induced by mesoscopic flow, squirt flow caused by cointype crack and squirt flow caused by pinch-type crack. While in the proposed model, those peaks are induced by squirt flow caused by coin-type crack, squirt flow caused by pinch-type crack and bubble linear vibration. Furthermore, it is obvious to observe that the mechanism of attenuation induced by two-phase fluid on patchysaturated model is important at seismic frequencies while the mechanism of attenuation induced by two-phase fluid in proposed model plays a significant role in vibrate frequencies. 


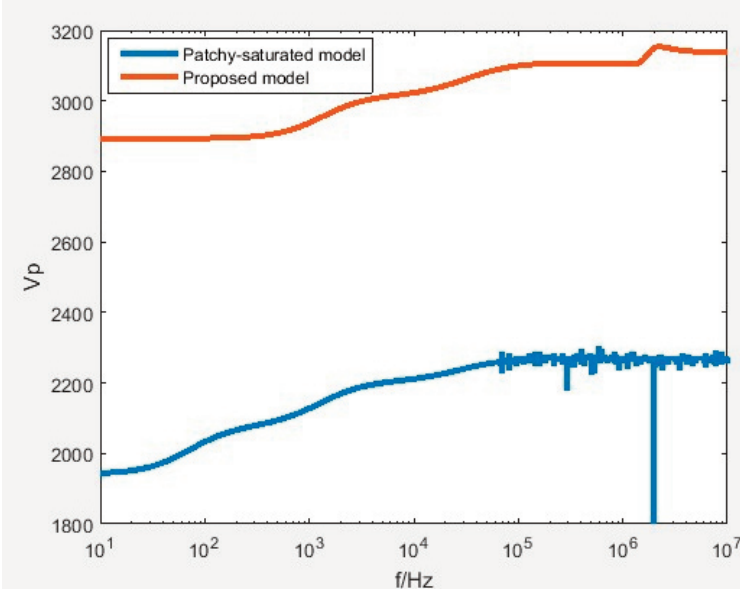

a)

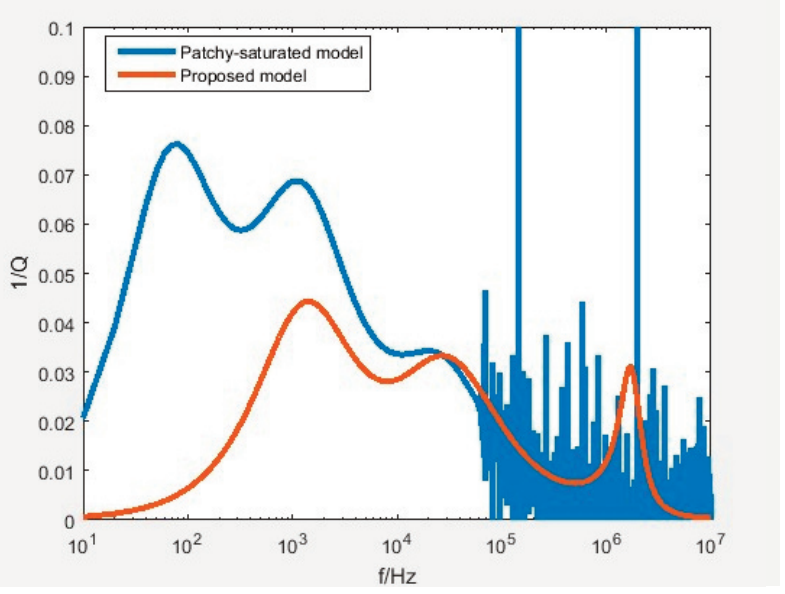

b)

Figure 5. The attenuation and dispersion of the $\mathrm{P}$ waves for patchy-saturated model and the proposed model. The dispersion velocity (a) and attenuation (b) for the P wave.

\subsection{Wave dispersion and attenuation characteristics and frequency dependent parameter}

For the sake of describing the dispersion and attenuation characteristics of the seismic wave more clearly, we compare the seismic wave dispersion and attenuation curves for different bubble fluid volumes (Figure 6), porosity (Figure 7), permeability (Figure 8), and fluid viscosity (Figure 9).

Figure 6 shows the dispersion and attenuation for the fast $\mathrm{P}$ wave and slow $\mathrm{P}$ wave for different bubble volume fraction in the frequency range of 10 to $10^{7} \mathrm{~Hz}$ with logarithmic scale. As shown in Figure 6, the bubble volume fraction only affects the seismic attenuation and dispersion caused by the linear vibration of the bubble. The velocity attenuation and the dispersion become obvious with increasing of the bubble volume fraction, which indicates that the amplitude of velocity attenuation increases with increasing of the bubble volume fraction. In a summary, bubble volume fraction is a frequency dependent parameter.

Figure 7 shows the dispersion and attenuation for the fast $\mathrm{P}$ wave and slow $\mathrm{P}$ wave for different porosity in the frequency range of 10 to $10^{7} \mathrm{~Hz}$ with logarithmic scale. As shown in Figure 7, the curves of fast P-wave dispersion change obviously with increasing of the porosity while the curves of slow P-wave dispersion do not change obviously with increasing of the porosity. With increase of the porosity, the attenuation caused by the "squirt-flow" and caused by the linear vibration of the bubble does not move while their amplitude reduces. Moreover, the curves of P-wave velocity and the peaks caused by the "Biot flow" move toward high frequencies with the increase of the porosity. In a summary, porosity is a frequency dependent parameter.

Figure 8 shows the dispersion and attenuation for the fast $\mathrm{P}$ wave and slow $\mathrm{P}$ wave for different permeability in the frequency range of 10 to $10^{7} \mathrm{~Hz}$ with logarithmic scale. As shown from the figure 8 , the bubble volume fraction only affects the seismic attenuation and dispersion caused by Biot mechanism. The curves of P-wave attenuation do not change obviously with increasing of the permeability. Furthermore, the curves of P-wave velocity and the peaks caused by the "Biot flow" move toward low frequencies with the decrease of the permeability. In a summary, permeability is a frequency dependent parameter.

Figure 9 shows the dispersion and attenuation for the fast $\mathrm{P}$ wave and slow $\mathrm{P}$ wave for different fluid viscosity in the frequency range of 10 to $10^{7} \mathrm{~Hz}$ with logarithmic scale. As shown in Figure 9, the curves of P-wave velocity and the peaks caused by the "Biot flow" move toward low frequencies with the increase of the fluid viscosity and the curves of P-wave velocity and the peaks caused by the "squirt flow" move toward low frequencies with the increase of the fluid viscosity. Both of the amplitude value reduced slightly. What attracts us most is the curves of $\mathrm{P}$-wave velocity and the amplitude of the peak caused by the linear vibration increases with the increase of the fluid viscosity, which proves that fluid viscosity is an important factor affecting attenuation caused by the linear vibration. In a summary, fluid viscosity is a frequency dependent parameter. 


\section{Yu Chen et al.}

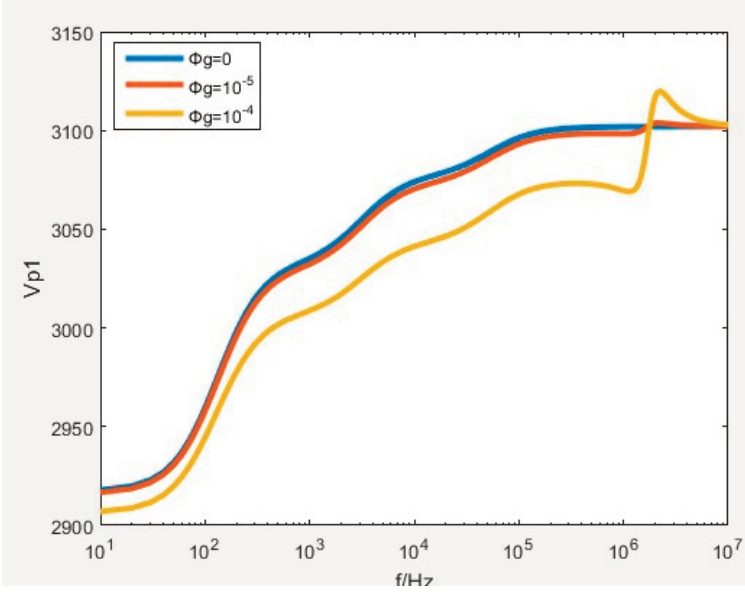

a)

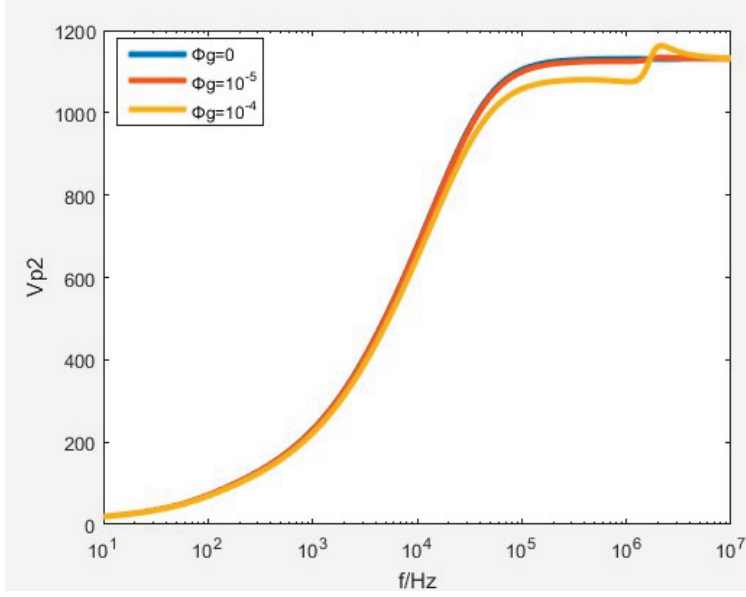

c)

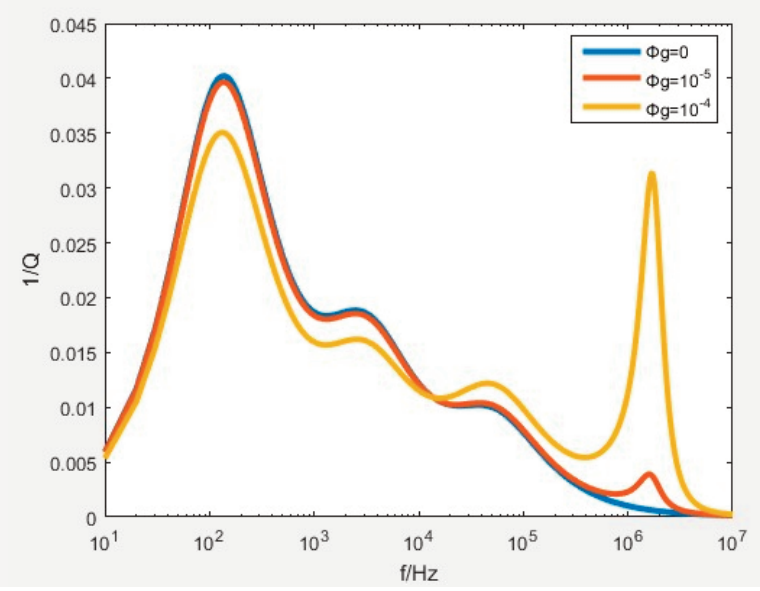

b)

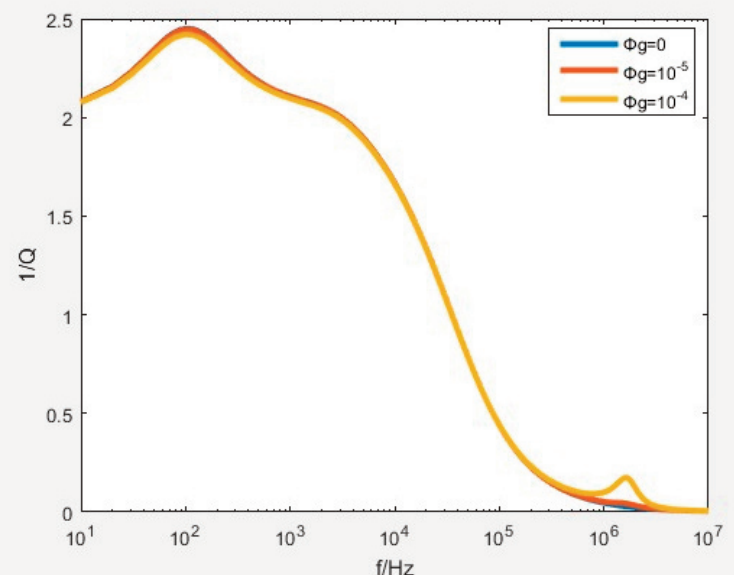

d)

Figure 6. The attenuation and dispersion of the fast and slow $\mathrm{P}$ waves for the porous medium containing different kinds of cracks and a small amount of bubbly fluid in the frame of Biot theory under condition of different bubbly fluid volume. The dispersion velocity (a) and attenuation (b) for the fast P wave, the dispersion velocity (c) and attenuation $(\mathrm{d})$ for the slow $\mathrm{P}$ wave. 


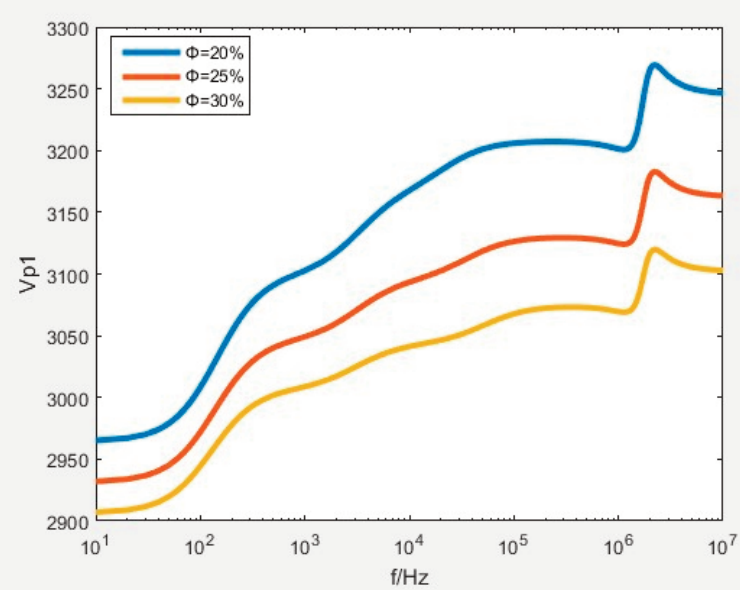

a)

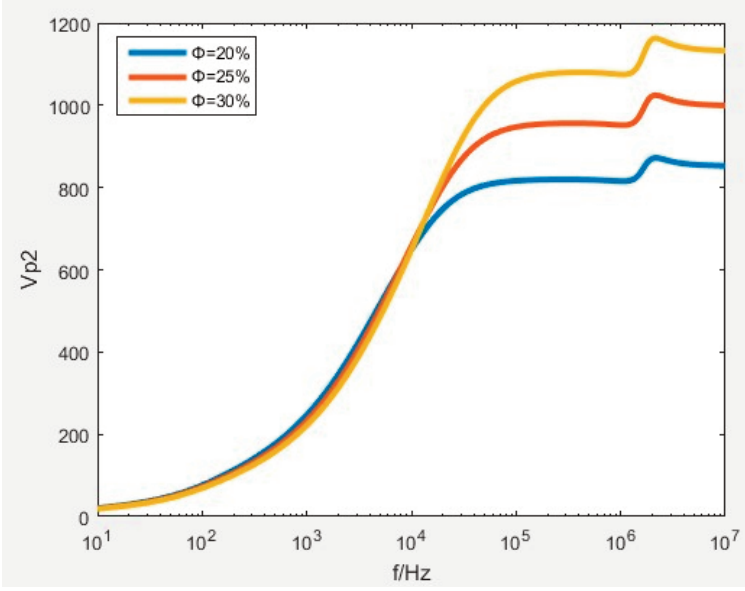

c)

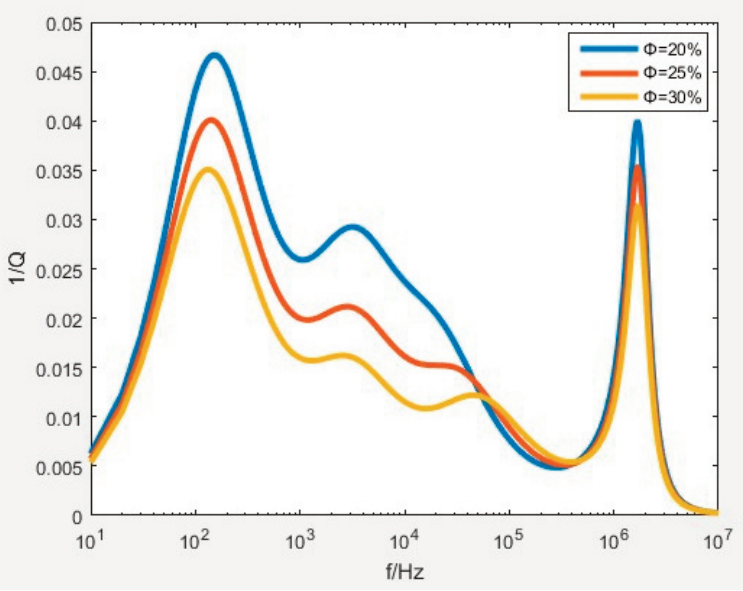

b)

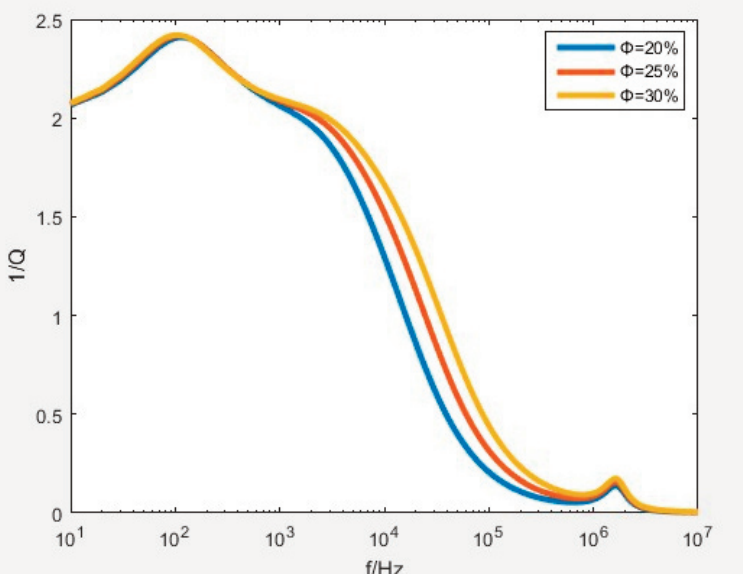

d)

Figure 7. The attenuation and dispersion of the fast and slow $\mathrm{P}$ waves for the porous medium containing different kinds of cracks and a small amount of bubbly fluid in the frame of Biot theory under condition of different porosity. The dispersion velocity (a) and attenuation (b) for the fast P wave, the dispersion velocity (c) and attenuation (d) for the slow P wave. 


\section{Yu Chen et al.}

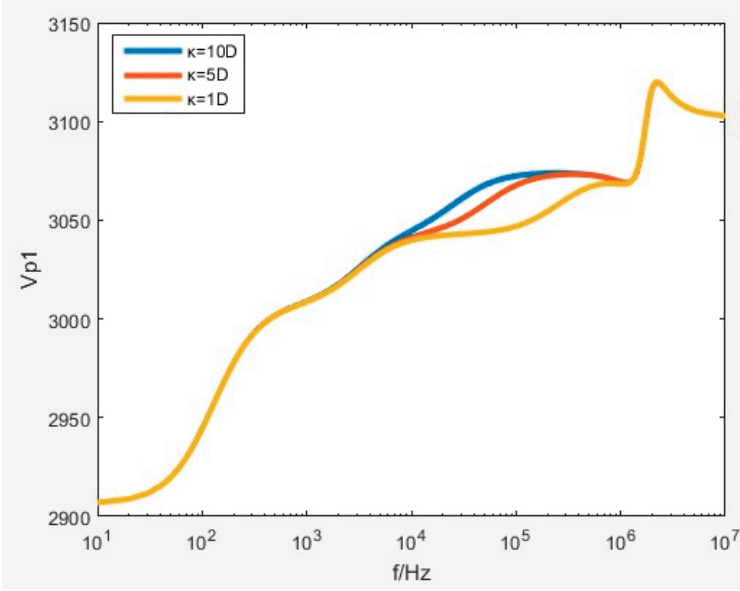

a)

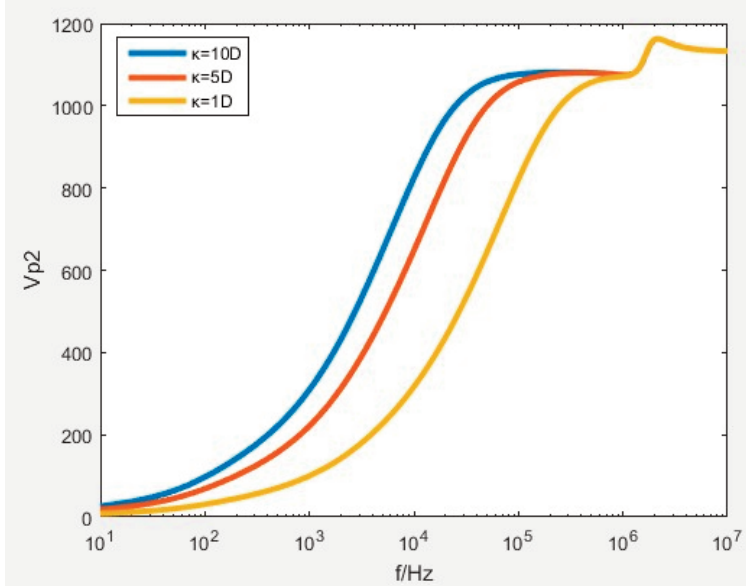

c)

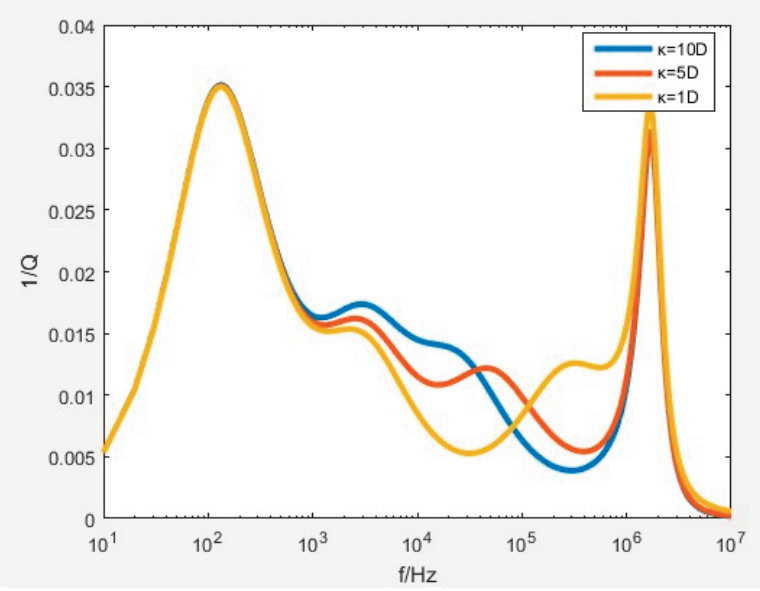

b)

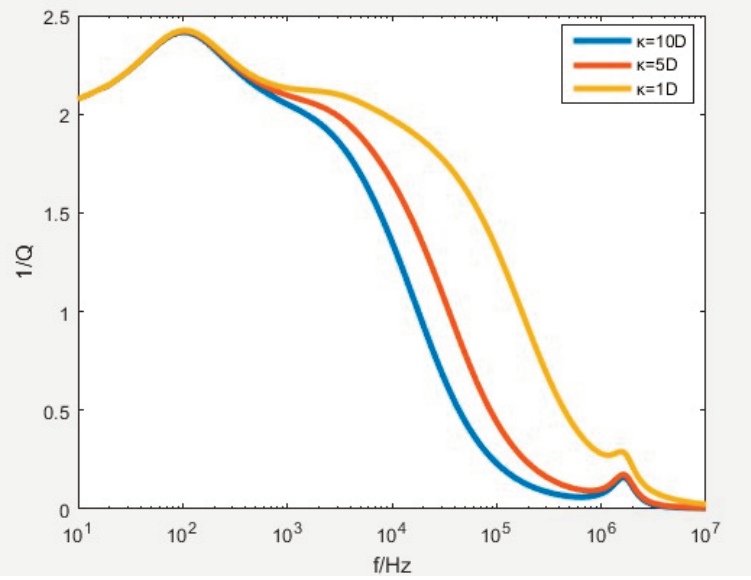

d)

Figure 8. The attenuation and dispersion of the fast and slow $\mathrm{P}$ waves for the porous medium containing different kinds of cracks and a small amount of bubbly fluid in the frame of Biot theory under condition of different permeability. The dispersion velocity (a) and attenuation (b) for the fast P wave, the dispersion velocity (c) and attenuation (d) for the slow P wave. 


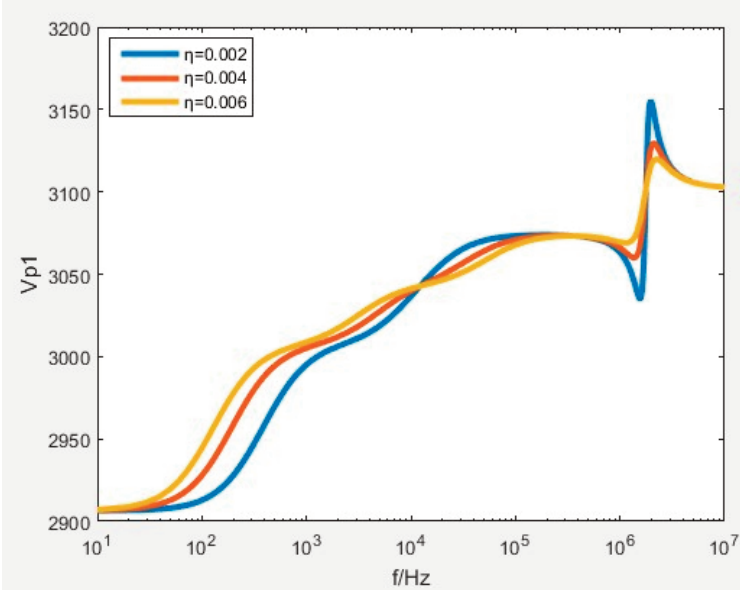

a)

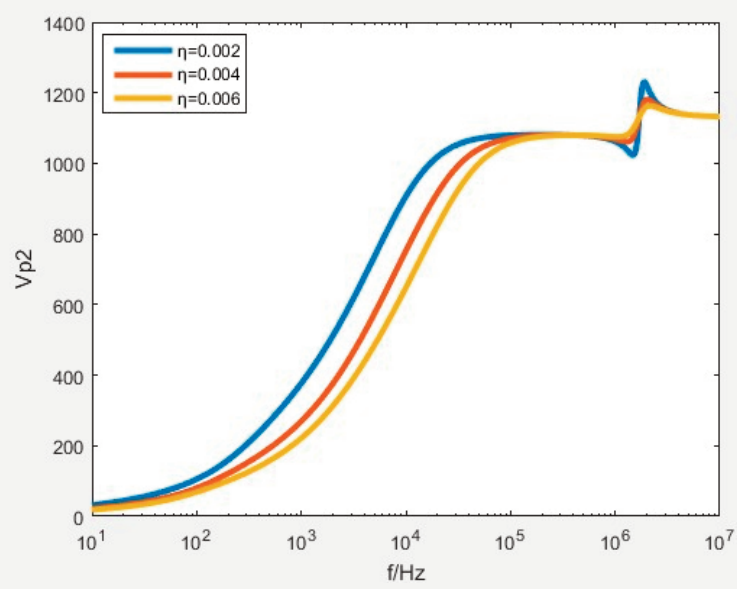

c)

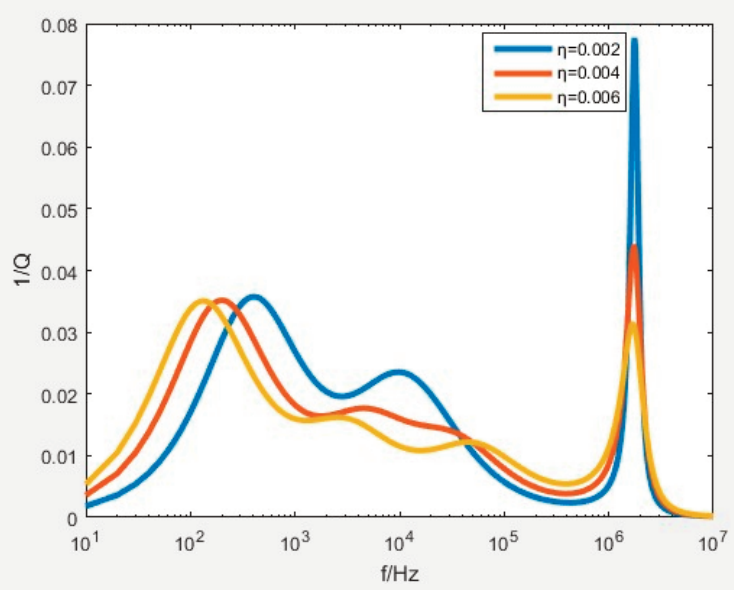

b)

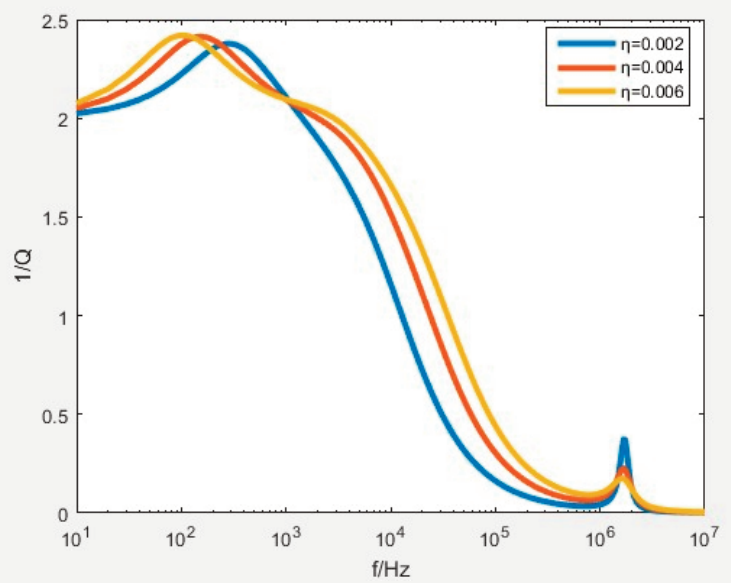

d)

Figure 9. The attenuation and dispersion of the fast and slow $\mathrm{P}$ waves for the porous medium including different kinds of cracks in the frame of Biot theory under condition of different fluid viscosity. The dispersion velocity (a) and attenuation (b) for the fast $\mathrm{P}$ wave, the dispersion velocity (c) and attenuation (d) for the slow $\mathrm{P}$ wave. 


\section{Yu Chen et al.}

\section{Conclusion}

Cracks and a small amount of bubbly fluid have an important influence on the propagation of elastic waves, which can produce considerable attenuation and dispersion of elastic wave propagation. Through the specific analysis of the squirt flow effect and the linear vibration of the bubble, we derive a theory of elastic wave that describes the porous medium containing various and a small amount of bubbly fluid. It can effectively simulate the influence of cracks and a small amount of bubbly fluid on elastic wave propagation and maintain the basic characteristics of Biot theory.

Acknowledgements. We would like to acknowledge the sponsorship of National Nature Science Foundation of China (41974119 U1762103), National Grand Project for Science and Technology (2016ZX05024004; 2017ZX05036005; 2017ZX05009001; 2017ZX05032003), National College Student Innovation and Entrepreneurship Training Program (201910425031), Science Foundation from Innovation and Technology Support Program for Young Scientists in Colleges of Shandong province and Young Elite Scientists Sponsorship Program by CAST (2017QNRC001).

\section{References}

Aki, K., and P. G. Richards (1980). Quantitative seismology: Theory and methods. W. H. Freeman \& Co.

Ba, J., J. M. Carcione, and J. X. Nie (2011). Biot-Rayleigh theory of wave propagation in double-porosity medi., J.G.R, 116, B06202, doi:10.1029/2010JB008185.

Ba, J., W. Xu, L. Y. Fu, J. M. Carcione, and L. Zhang (2017). Rock anelasticity due to patchy saturation and fabric heterogeneity: A double double-porosity model of wave propagation. J.G.R., 122, 1949-1976, doi:10.1002/2016JB013882.

Biot, M. A. (1956a). Theory of propagation of elastic waves in fluid-saturated porous solid - Part 1: Low frequency range. J. Acoust. Soc. Am., 28, 168-178, doi:10.1121/1.1908239.

Biot, M. A. (1956b). Theory of propagation of elastic waves in a fluid-saturated porous solid: Part II - Higher frequency range. J. Acoust. Soc. Am., 28, 179-191, doi:10.1121/1.1908241.

Biot, M. A. (1962). Mechanics of deformation and acoustic propagation in porous media. J. Appl. Phys., 33, 14821498, doi:10.1063/1.1728759.

Dutta, N. C., and H. Odé (1979a). Attenuation and dispersion of compressional waves in fluid-filled porous rocks with partial gas saturation (White model): Part I - Biot theory. Geophysics, 44, 1777-1788, doi:10.1190/1.1440938.

Dutta, N. C., and H. Odé (1979b). Attenuation and dispersion of compressional waves in fluid filled porous rocks with partial gas saturation (White model): Part II Results. Geophysics, 44, 1789-1805, doi:10.1190/1.1440939.

Dvorkin, J., and A. Nur (1993). Dynamic poroelasticity: A unified model with the squirt and the Biot mechanisms. Geophysics, 58, 524-533, doi:10.1190/1.1443435.

Dvorkin, J., N. H. Richard, and A. Nur (1994). The squirt-flow mechanism: Macroscopic description. Geophysics, 59, 428-438, doi:10.1190/1.1443605.

Dvorkin, J., G. Mavko, and A. Nur (1995). Squirt flow in fully saturated rocks. Geophysics, 60, 97-107, doi:10.1190/1.1443767.

Keller, J. B., and M. J. Miksis (1980). Bubble oscillations of large amplitude. J. Acoust. Soc. Am, 68, 628-633, doi:10.1121/1.384720.

Kumar, M., M. S. Barak, and M. Kumari (2019). Reflection and refraction of plane waves at the boundary of an elastic solid and double-porosity dual-permeability materials. Pet. Sci., 16, 298-317, doi:10.1007/s12182-018-0289-z.

Mavko, G., and D. Jizba (1991). Estimating grain-scale fluid effects on velocity dispersion in rocks. Geophysics, 56, 1940-1949, doi:10.1190/1.1443005.

Mavko, G., and A. Nur (1979). Wave propagation in partially saturated rocks. Geophysics, 44, 161-178, doi:10.1190/1.1440958.

Mavko, G., T. Mukerji, and J. Dvorkin (2009). The rock physics handbook, $2^{\text {nd }}$ ed. Cambridge University Press.

Murphy, W. F., K. W. Winkler, and R. L. Kleinberg (1986). Acoustic relaxation in sedimentary rocks: Dependence on grain contacts and fluid saturation. Geophysics, 51, 757-766, doi:10.1190/1.1442128. 
Müller, T. M., B. Gurevich, and M. Lebedev (2010). Seismic wave attenuation and dispersion resulting from waveinduced flow in porous rocks. A review. Geophysics, 75, no. 5, A147-A164, doi:10.1190/1.3463417.

Parra, J. O. (1997). The transversely isotropic poroelastic wave equation including the Biot and the squirt mechanisms: Theory and application. Geophysics, 62, 309- 318, doi:10.1190/1.1444132.

Pride, S. R., and J. G. Berryman (2003a). Linear dynamics of double-porosity and dual-permeability materials. Part 1: Governing equations and acoustic attenuation. Phys. Rev. E, 68, 036603, doi:10.1103/PhysRevE.68.036603.

Pride, S. R., and J. G. Berryman (2003b). Linear dynamics of double-porosity and dual-permeability materials: Part II - Fluid transport equations. Phys. Rev. E, 68, 036604, doi:10.1103/PhysRevE.68.036604.

Pride, S. R., J. G. Berryman, and J. M. Harris (2004). Seismic attenuation due to wave induced flow. J. G. R., 109, B01201, doi:10.1029/2003JB002639.

Pride, S. R. (2005). Relationships between seismic and hydrological properties: Hydrogeophysics, 50, 253-290, doi:10.1007/1-4020-3102-5 9.

Sun, W., F. Xiong, J. Ba, and J. M. Carcione (2018). Effects of ellipsoidal heterogeneities on wave propagation in partially-saturated double-porosity rocks: Geophysics, 83, no.3, WC71-WC81, doi:10.1190/GEO2017-0549.1.

Tang, X. M., and C. H. Cheng (2004). Quantitative borehole acoustic methods: Elsevier Science Publishing Co. Oxford.

Tang, X. (2011). A unified theory for elastic wave propagation through porous media containing cracks - An extension of Biot's poroelastic wave theory. Sci. China Earth Sci., 54, 1441-1452, doi:10.1007/s11430-011-4245-7.

Tang, X. M., X. L. Chen, and X. K. Xu (2012). A cracked porous medium elastic wave theory and its application to interpreting acoustic data from tight formations. Geophysics, 77, no. 6, D245-D252, doi:10.1190/geo2012-0091.1.

Vogelaar, B., and D. Smeulders (2007). Extension of White's layered model to the full frequency range: Geophysical Prospecting, 55, 685-695, doi:10.1111/j.1365-2478.2007.00648.x.

Vogelaar, B., D. Smeulders, and J. Harris (2010). Exact expression for the effective acoustics of patchy-saturated rocks. Geophysics, 75, no. 4, N87-N96, doi:10.1190/1.3463430.

Wang, Z., and A. Nur (1998). Dispersion analysis of acoustic velocities in rocks. J. Acoust. Soc. Am., 87, 2384-2395, doi:10.1121/1.399551.

Wang, T., Z. W. Cui, J. X. Liu, and K. X. Wang (2018). Propagation of elastic waves in saturated porous medium containing a small amount of bubbly fluid: Acta Phys. Sin. (in Chinese), 67, 114301, doi:10.7498/aps.67.20180209.

White, J. E. (1975). Computed seismic speeds and attenuation in rocks with partial gas saturation. Geophysics, 40, 224-232, doi:10.1190/1.1440520.

Wu, J. L., G. C. Wu, and Z. Y. Zong (2015). Attenuation of P waves in a porous medium containing various cracks. Chinese J. Geophys. -CH., 58, 1378-1389, doi:10.6038/cjg20150424.

Zhang, L., J. Ba, J. M. Carcione, and W. Sun (2019). Modeling wave propagation in cracked porous media with pennyshaped inclusions: Geophysics, 84, no. 4, WA141-WA151, doi:10.1190/geo2018-0487.1.

Zhao, Z., X. Yin, and Z. Zong (2018). Analytical seismic wave attenuation and velocity dispersion in layered doubleporosity media: Ann. of Geophys., 61, SE343, doi:10.4401/ag-7649. 